\title{
Ablation of Ventricular Tachycardia in Patients with Nonischemic Cardiomyopathy
}

\author{
FRANK BOGUN, M.D. and FRED MORADY, M.D. \\ From the Division of Cardiology, University of Michigan Health System, Ann Arbor, Michigan, USA
}

\begin{abstract}
Nonischemic Cardiomyopathy. Mapping and ablation of ventricular tachycardia (VT) in patients with nonischemic cardiomyopathy can be challenging. In this article, we describe the approach to VT in patients with nonischemic cardiomyopathy that we use at the University of Michigan. Imaging, especially with delayed enhanced cardiac magnetic resonance imaging, plays a central role in planning a mapping and ablation procedure. During the mapping procedure, pace-mapping in conjunction with voltage maps focusing on areas with isolated potentials helps to identify scar areas that are critical for VT in these patients. (J Cardiovasc Electrophysiol, Vol. 19, pp. 1227-1230, November 2008)
\end{abstract}

ventricular tachycardia, ablation, nonischemic cardiomyopathy

\section{Introduction}

In this article, we describe the approach used at the University of Michigan to perform mapping and ablation procedures in patients with nonischemic cardiomyopathy.

Nonischemic cardiomyopathy is not a single disease entity. Cardiac sarcoidosis, hypertrophic cardiomyopathy, amyloidosis, valvular heart disease, and arrhythmogenic right ventricular dysplasia all need to be considered as possible etiologies for nonischemic cardiomyopathy. The single-best screening test is cardiac magnetic resonance imaging (MRI) with delayed enhancement (DE-MRI). It is preferable to perform the MRI before implantation of an implantable cardioverter defibrillators (ICD).

\section{Patient Selection}

Patients with a nonischemic cardiomyopathy typically qualify for implantation of an ICD for either $1^{\circ}$ or $2^{\circ}$ prevention of sudden death. If a patient receives appropriate ICD shocks that are frequent enough to interfere with quality of life, we usually institute antiarrhythmic drug therapy to reduce the number of appropriate shocks. At the University of Michigan, the most common indication for catheter ablation of ventricular tachycardia (VT) in patients with nonischemic cardiomyopathy is frequent ICD discharges (or electrical storm) that are refractory to antiarrhythmic drug therapy.

In patients with nonischemic cardiomyopathy who present with a first documented episode of sustained, monomorphic VT and who have not already received an ICD, we may offer catheter ablation as a therapeutic option in conjunction

Address for correspondence: Frank Bogun, M.D., Division of Cardiology, University of Michigan Health System, 1500 East Medical Center Drive SPC 5853, Ann Arbor, MI 48109-5853, USA. Fax: 734-936-4027; E-mail: fbogun@med.umich.edu

Manuscript received 10 March 2008; Revised manuscript received 2 June 2008; Accepted for publication 11 June 2008.

doi: $10.1111 / \mathrm{j} .1540-8167.2008 .01268 . \mathrm{x}$ with ICD implantation. Some patients may prefer to undergo catheter ablation in the hope of avoiding future ICD shocks. But because of the future risk of life-threatening VT due to progression of the myopathic process, we do not consider catheter ablation to be a substitute for an ICD, even when excellent short-term results are achieved. This especially is true in patients with cardiac sarcoidosis, even when left ventricular function is normal. In patients with cardiac sarcoidosis, we generally reserve catheter ablation for patients who have failed to respond not only to antiarrhythmic drugs, but also to immunosuppressive therapy.

It is important to keep in mind that frequent premature ventricular contractions (PVCs) may cause a reversible form of dilated nonischemic cardiomyopathy. In patients who present with a dilated cardiomyopathy of unclear etiology and who have a PVC burden $>15-20 \%$, catheter ablation of the PVC's is appropriate prior to ICD implantation for $1^{\circ}$ prevention of sudden death. Successful elimination of the PVCs may result in improvement in left ventricular function, ${ }^{1}$ such that the patient no longer qualifies for an ICD.

In 2007, we performed ablation procedures for VT in 16 patients with nonischemic cardiomyopathy and in 19 patients with frequent PVCs and cardiomyopathy.

\section{Preablation Work-Up}

A pre-procedure MRI with delayed enhancement is useful because it demonstrates the extent and location of the scar tissue that may harbor the arrhythmia substrate (Fig. 1). This helps to select the most appropriate mapping approach: right ventricular versus left ventricular, and endocardial versus epicardial. In our experience, scar often is demonstrated by delayed enhancement during MRIs, especially in the presence of inducible VT. The location of the scar tissue often corresponds to areas critical for VT circuits in these patients. Unfortunately, most patients presenting with appropriate ICD shocks currently cannot undergo MRIs because of the device.

All available 12-lead electrocardiograms and ICD stored electrocardiograms of the VT(s) should be reviewed before 


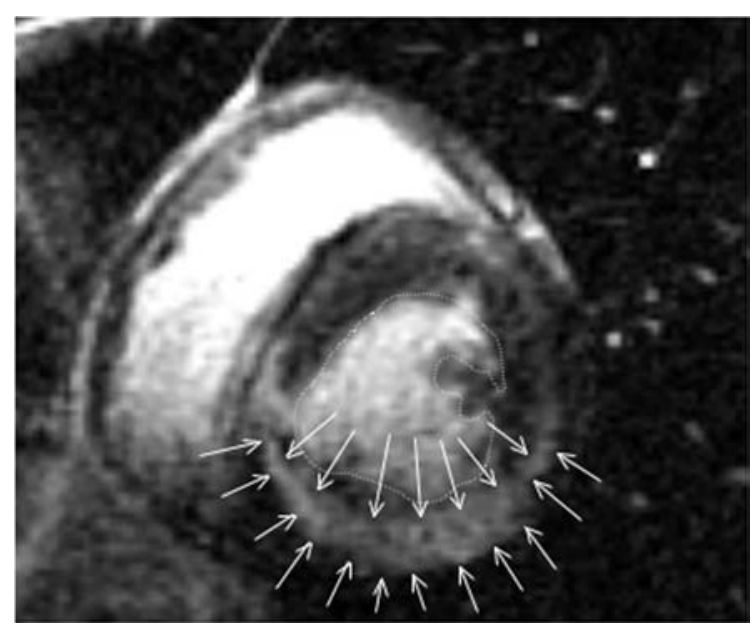

Figure 1. Short axis view of a delayed-enhanced magnetic resonance image in a patient with nonischemic cardiomyopathy. There is predominantly intramural and epicardial uptake of gadolinium indicative of scar tissue (arrows). The endocardium (outlined by a dotted line) is relatively preserved with the exception of two areas at the 9 and 1 o'clock position where the endocardium also takes up contrast. Endocardial ablation of VT was unsuccessful in this patient. (Epicardial ablation was not performed in this patient.)

the ablation procedure. Before the ablation procedure, a left ventricular thrombus should be ruled out by transthoracic echocardiography or DE-MRI.

\section{Selection of Target Sites for Ablation}

Reentry is the most common mechanism of $\mathrm{VT}^{2}$ and therefore, a mapping strategy similar to postinfarction VT usually can be used (Fig. 2). ${ }^{3}$ Entrainment mapping, however, can only be performed for hemodynamically tolerated VTs. In our experience, about one-third of patients have tolerated VTs that can be mapped with entrainment mapping. During the procedure, multipolar catheters are positioned in the high right atrium, the His bundle position, and the right ventricular apex. The main reason for the His bundle catheter is to look for bundle branch reentry tachycardia. The right ventricular catheter is positioned at the apex, and the postpacing interval at this site also is helpful in diagnosing bundle branch reentry tachycardia.

We perform programmed right ventricular stimulation with 1-4 extrastimuli to induce VT. An electromagnetic map- ping system (CARTO, Biosense, Diamond Barr, CA, USA) is used to aid mapping and navigation. An irrigated-tip catheter (Thermocool, 3.5-mm tip electrode separated by $1 \mathrm{~mm}$ from a 2-mm ring electrode) is used for mapping and ablation.

Endocardial activation and/or entrainment mapping is performed during VT if the VT is tolerated hemodynamically, This is possible in less than $50 \%$ in our experience. For VTs resulting in hemodynamic compromise, pace-mapping and voltage-mapping are performed simultaneously. Our voltage maps contain about 200-500 mapping points depending on the presence or absence of low-voltage areas and sites with isolated potentials. At other institutions, cardiopulmonary support has been used effectively to allow mapping of nontolerated VTs.

As each point is collected for the voltage map, pacing is performed at sites with low-voltage (bipolar voltage amplitude of $<1.5 \mathrm{mV}$ ) or an isolated potential. Isolated potentials are helpful in postinfarction patients to guide radiofrequency ablation. It appears that they are equally or even more helpful in nonischemic cardiomyopathy patients. A good pace-map (defined as $\geq 10 / 12$ matching leads between the pace-map and the targeted VT) at a site where there is an isolated potential is useful for identifying a critical component of the reentry circuit. (Fig. 3) A good pace-map at a site without an isolated potential is a less specific indicator of a critical site for ablation.

Isolated potentials reflect fixed scar tissue. In our experience, they can be identified in approximately $50 \%$ of patients undergoing mapping and ablation of VT in the setting of nonischemic cardiomyopathy. It is possible that fixed scar tissue plays less of a role and that functional areas of block are more prevalent in patients in whom isolated potentials cannot be identified. This may have important implications since focal ablation procedures are less likely to eliminate their VTs. Patients devoid of isolated potentials in our experience appear to have a less favorable outcome with respect to ablation. However, although none may be present on the endocardium, isolated potentials may be found during epicardial mapping.

Some patients do not have isolated potentials or detectable scar tissue at either endocardial or epicardial locations. In these patients, the scar tissue may be intramural and ablation does not appear to yield satisfactory results, especially if the VT is pleomorphic.

If endocardial mapping does not yield an effective ablation site, transcutaneous puncture to enter the pericardial space is performed at a separate session. In most of these
Figure 2. An effective ablation site in a patient with nonischemic cardiomyopathy. There is concealed entrainment and a diastolic potential during VT. The electrogram-QRS interval matches the stimulus-QRS interval (both are $210 \mathrm{~ms}$ ). Shown are leads I, II, III, VI, and V6 and the intracardiac tracings from the mapping catheter (Map). Pacing cycle length is $450 \mathrm{~ms}$ and the VT cycle length is 490 ms.

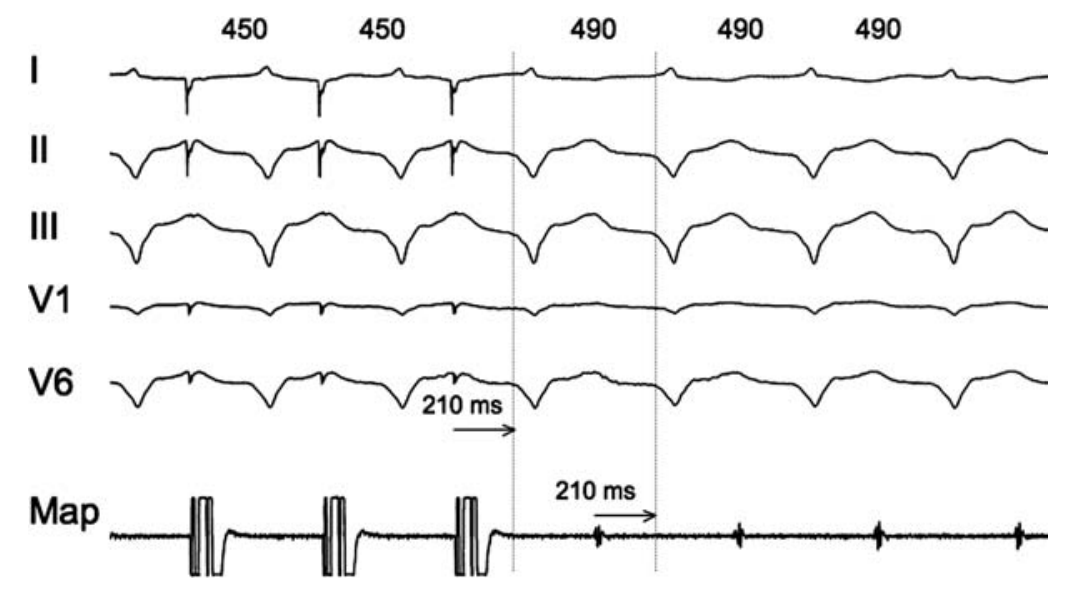




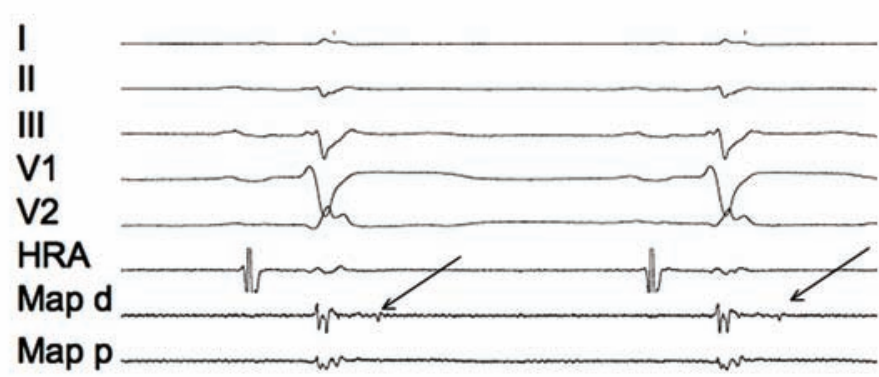

$200 \mathrm{~ms}$

B

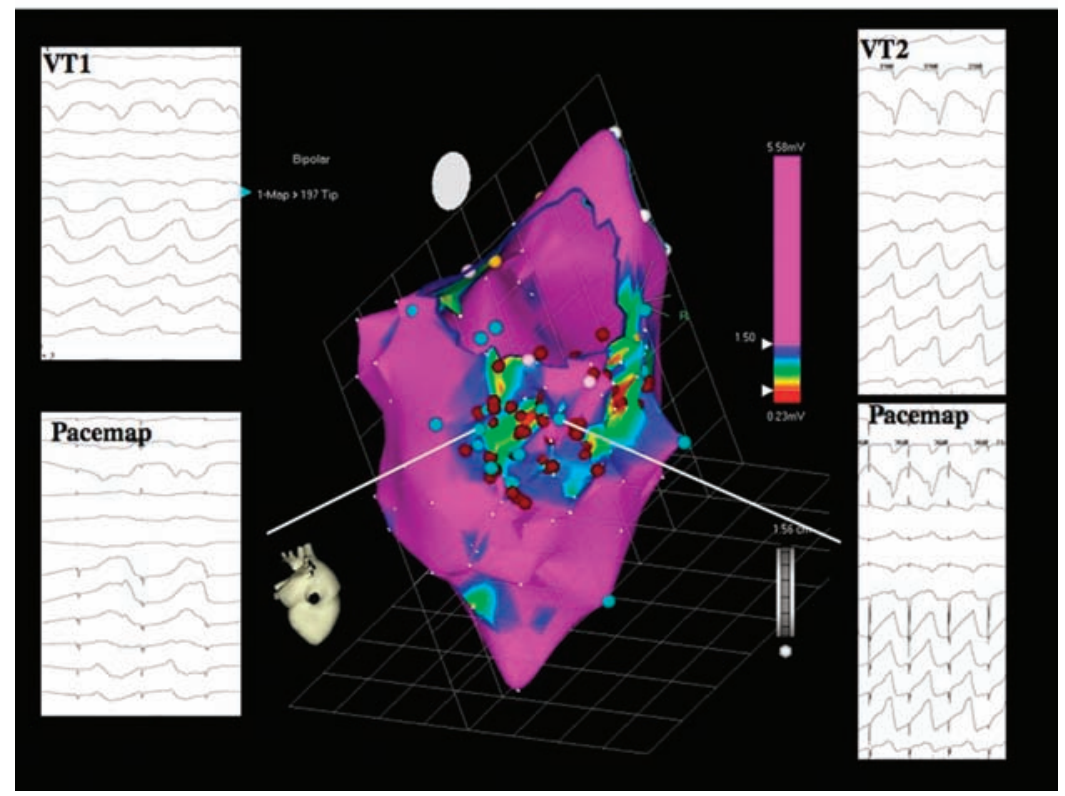

Figure 3. (A) This figure illustrates isolated potentials during sinus rhythm and pace-mapping. Leads I, II, III, and V2 and intracardiac electrograms from the high right atrium (HRA) and the distal and proximal poles of the mapping catheter (MAP $d$ and Map $p$ ) are displayed. The mapping catheter was positioned at the posterobasal left ventricle. (B) A voltage map of the posterobasal left ventricle. Pace-mapping was performed at sites with isolated potentials. There were two adequate pace-maps at sites with isolated potentials. Inserts on the left indicate the electrocardiogram of the targeted VT 1 (top left) and the corresponding pace-map (bottom left) at a site with an isolated potential (shown in Fig. 1A). On the right is VT 2 (top right) with a matching pacemap (bottom right) also at a site with an isolated potential. patients, limited epicardial mapping inside the coronary venous system demonstrates early activation during VT at the epicardium. If pace-mapping demonstrates a suitable ablation site within the coronary venous system, radiofrequency ablation is attempted within the vein before proceeding with mapping within the pericardial space. We have found that an epicardial approach is fruitful in about $30 \%$ of patients with nonischemic cardiomyopathy and VT.

An option to the stepwise approach is to enter the pericardial space during the first ablation procedure, before the administration of heparin. This may be appropriate if there are clues on the electrocardiogram, that suggest an epicardial VT or if there is documentation by MRI of largely epicardial scar. The epicardial procedure is performed as described by Sosa et al. ${ }^{4}$ After the pericardial space has been accessed with a guide wire, an 8-F introducer sheath is inserted.

\section{Ablation Technique and Endpoints}

Radiofrequency energy is delivered to the endocardium using a 3.5-mm open irrigated catheter (Thermocool, CARTO, Biosense). The power setting is adjusted to an impedance drop of $10 \Omega$ starting with $30-35 \mathrm{~W}$. If radiofrequency energy is applied during VT, it is applied for $30 \mathrm{sec}-$ onds and, if VT does not terminate, the catheter is moved to an alternate site. If VT terminates during the energy application, the application is continued for a total of 120 seconds at this site. In the setting of nontolerated VTs, when pace-mapping is used, radiofrequency energy is delivered for $60-120 \mathrm{~ms}$ at a site meeting ablation criteria, then the catheter is moved to an alternate site.

A 4-mm tip catheter (Navistar CARTO, Biosense) is used for epicardial-mapping. Prior to an epicardial ablation, a coronary angiogram is obtained to ensure a distance of at least $1.0 \mathrm{~cm}$ from a major coronary artery. For smaller branches, a distance of $0.5 \mathrm{~cm}$ from the ablation catheter is probably safe. Radiofrequency energy is delivered at an initial power of 10-15 W and the power is titrated to achieve a target temperature of $55^{\circ} \mathrm{C}$. The duration of the radiofrequency energy application is the same as described above for endocardial ablation. The effect of the ablation lesions is assessed by pacing at an output of $10 \mathrm{~mA}$ at a pulse width of $2 \mathrm{~ms}$. If there is still capture, an 8-mm tip catheter (Navistar CARTO, Biosense) or an internally irrigated catheter with a 4-mm tip is used (Boston Scientific Inc, Natick, MA, USA). Also, high-output pacing is performed to assess for phrenic nerve capture.

If radiofrequency energy is delivered in the coronary venous system, we use an irrigated tip catheter at an initial 
power of $15 \mathrm{~W}$, titrating up to achieve a $10 \Omega$ impedance drop.

The end point of the procedure is noninduciblity of all the VTs for which an appropriate target site was identified. We attempt to target all VTs, although it is unclear in the setting of nonischemic cardiomyopathy whether targeting all inducible VTs is superior to targeting only the VTs that have been clinically documented. Noninducibility of all VTs is achieved in about $50-70 \%$ of patients.

\section{Outcomes of Ablation}

Radiofrequency ablation helps to control VTs refractory to medical treatment in most patients $(60-70 \%)$ with nonischemic cardiomyopathy. If patients were treated with antiarrhythmic medications before the ablation procedure, treatment with these medications is continued as tolerated postablation.

Patients who fail to respond to catheter ablation and who continue having frequent episodes of VT may qualify for a left ventricular assist device or heart transplantation. This approach was necessary in two patients within the last 2 years at the University of Michigan.

\section{Avoiding Complications}

Given the left ventricular dysfunction in patients with nonischemic cardiomyopathy, when using an open irrigated tip catheter during an ablation procedure, it is important to monitor fluid status carefully during the procedure. A Foley catheter helps to adequately monitor urine output and the need for intravenous diuretics to avoid pulmonary edema during the procedure.

The mapping and ablation procedure is performed under conscious sedation with propofol or etomidate administered by an anesthesiologist or nurse anesthetist. The same agents are used for epicardial-mapping procedures; however, the level of sedation is deeper during access of the pericardial space, to avoid patient movement. General anesthesia may further lower the blood pressure that often is already low in the nonsedated state. Furthermore, general anesthesia may suppress the inducibility of VT. If general anesthesia is used during an epicardial-mapping and ablation procedure, muscle paralysis should be avoided since diaphragmatic capture might be absent when high-output pacing is performed in the vicinity of the phrenic nerve. During epicardial ablation, caution should be exerted to avoid coronary artery and phrenic nerve injury. We routinely perform coronary angiograms in multiple planes prior to ablation with the catheter located at the selected ablation site. Also, high-output pacing is performed from the tip of the ablation catheter at an output of $20 \mathrm{~mA}$ at a pulse width of $2 \mathrm{~ms}$ to look for phrenic nerve capture.

Our complication rate in patients with nonischemic cardiomyopathy is no different compared to patients with ischemic cardiomyopathy. Less than 5\% of patients have major complications, such as cardiac tamponade, thromboembolic events, or death. Electromechanical dissociation after multiple VT inductions can occur in patients with very low ejection fractions. Limiting the number of inductions of VT may help to prevent this life-threatening complication. One patient with frequent ICD discharges died 4 days after the mapping and ablation procedure with worsening congestive heart failure. This patient already required inotropic support prior to the ablation, and it is uncertain whether the patient's death was related to the ablation procedure.

\section{References}

1. Bogun F, Crawford T, Reich S, Koelling TM, Armstrong W, Good E, Jongnarangsin K, Marine JE, Chugh A, Pelosi F, Oral H, Morady F: Radiofrequency ablation of frequent, idiopathic premature ventricular complexes: Comparison with a control group without intervention. Heart Rhythm 2007;4:863-867.

2. Soejima K, Stevenson WG, Sapp JL, Selwyn AP, Couper G, Epstein LM: Endocardial and epicardial radiofrequency ablation of ventricular tachycardia associated with dilated cardiomyopathy: The importance of low-voltage scars. J Am Coll Cardiol 2004;43:1834-1842.

3. Bogun F, Kim HM, Han J, Tamirissa K, Tschopp D, Reich S, Elmouchi D, Igic P, Lemola K, Good E, Oral H, Chugh A, Pelosi F, Morady F: Comparison of mapping criteria for hemodynamically tolerated, postinfarction ventricular tachycardia. Heart Rhythm 2006;3:20-26.

4. Sosa E, Scanavacca M, D'Avila A, Pilleggi F: A new technique to perform epicardial mapping in the electrophysiology laboratory. J Cardiovasc Electrophysiol 1996;7:531-536. 